Economía, Sociedad y Territorio, vol. xx, núm. 64, 2020, 787-811.

DOI: https://doi.org/10.22136/est20201621

\title{
Tipo de vivienda, barreras físicas y sensación de inseguridad en la colonia
}

\section{Type of housing, physical barriers and feeling of insecurity in the neighborhood}

\author{
Carlos Vilalta* \\ Lucía Carmina JaSSO** \\ Gustavo Fondevila***
}

\begin{abstract}
In this study we analyze whether the type of housing and the physical barriers placed between the street and the entrance of the street hold a statistical relationship with the feeling of insecurity in the neighborhood. The results suggest rejecting such a hypothesis given the lack of evidence. This research allows the reader to know the correlates associated with the feeling of insecurity in the neighborhood. For this study, bivariate tests and multivariate models were used. Data were collected from the National Survey of Victimization and Perception of Public Safety.
\end{abstract}

Keywords: insecurity, fear of crime, housing, neighborhood, Mexico.

\section{Resumen}

En este estudio analizamos si el tipo de vivienda y las barreras físicas colocadas entre la calle y la entrada de la misma mantienen una relación estadística con la sensación de inseguridad en el entorno de la colonia. Los resultados sugieren rechazar tal hipótesis por no existir evidencia. Esta investigación permite al lector conocer los correlativos que se asocian con la sensación de inseguridad en la colonia. Para la realización de este análisis se utilizaron pruebas bivariadas y modelos multivariados; asimismo, se recabaron datos de la Encuesta Nacional de Victimización y Percepción sobre Seguridad Pública.

Palabras clave: inseguridad, miedo al crimen, vivienda, colonia, México.

* Centro de Investigación en Ciencias de Información Geoespacial, A. C, correo-e: cvilalta@ centrogeo.edu.mx

**Instituto de Investigaciones Sociales de la Universidad Nacional Autónoma de México, correoe: carmina.jasso@sociales.unam.mx

*** Centro de Investigación y Docencia Económica, correo-e: gustavo.fondevila@cide.edu 


\section{Introducción}

El estudio científico de la sensación de inseguridad en el ámbito de la colonia o vecindario tiene una larga historia en general, aunque no rebasa las tres décadas de historia en México. El enfoque con el cual se ha tratado el tema consiste en dilucidar cómo influye el contexto socioespacial del individuo en su sensación de inseguridad, de tal modo que muchos estudios se han centrado en analizar las condiciones de las colonias y las circunstancias familiares y sociales de los individuos; incluso, han surgido diversas teorías al respecto, tales como las de incivilidad, vulnerabilidad física y social y redes sociales. Sin omitir que otras investigaciones, con un gran peso teórico, advierten sobre la experiencia de victimización delictiva, ya que ésta deja un efecto psicológico en la víctima de una mayor alerta y sensación de inseguridad.

Motivados por estudios previos, por las brechas de conocimiento existentes con respecto al tema, así como por el problema generalizado de la inseguridad en México, el enfoque de este análisis radica en responder si el tipo de vivienda y las barreras físicas colocadas a la entrada de la misma tienen alguna relación con la sensación de inseguridad en el entorno de la colonia. Al iniciar este estudio la hipótesis planteada consistía en verificar quiénes eran más proclives a sentirse inseguros: si los residentes de departamentos en su colonia o los residentes de casas solas. Igualmente, para establecer las variables de estudio consideramos si aquellas personas que colocan barreras físicas entre la calle y la entrada a sus viviendas también mostrarían una mayor proclividad a sentirse inseguros en sus colonias de residencia, considerando que aquellos que viven más protegidos y aislados de su entorno son los que se sienten más inseguros. No obstante, los resultados de este estudio sugieren rechazar tal hipótesis por no existir evidencia para comprobarla. Por el contrario, el análisis desarrollado en este estudio indica que no es el tipo de vivienda ni las barreras físicas colocadas a la entrada del domicilio las que propician la sensación de inseguridad en la colonia, sino que son otros correlativos los que se asocian significativamente con dicha sensación en este entorno.

Este estudio se divide en cuatro partes; en la primera de ellas se revisaron los estudios previos, en donde hacemos una distinción de correlativos relacionados con el tipo de vivienda y correlativos relacionados con la colonia. En la segunda parte presentamos la metodología de estudio, en donde detallamos la fuente de información utilizada, las variables dependientes e independientes, las variables de control y el análisis estadístico. En la tercera parte exponemos los resultados del análisis estadístico. En el cuarto apartado presentamos la discusión y las conclusiones, en donde 
hacemos énfasis tanto en las implicaciones de los hallazgos como en las limitaciones del estudio.

\section{Estudios previos}

\subsection{Sobre los tipos de viviendas}

En la literatura científica proliferan los estudios sobre la sensación de inseguridad desde diferentes enfoques teóricos y cada vez se ha profundizado más en las distintas variables que lo explican y sus efectos sociales, económicos, culturales, entre otros. Si bien cada cuerpo teórico parte de premisas diferentes, coinciden en que esta sensación de inseguridad no ocurre en el vacío (Ceccato y Wilhelmsson, 2011), y que además de los factores individuales y sociales también son relevantes las condiciones y dinámicas del vecindario (Wyant, 2008).

Las viviendas son lugares en donde se encierran las ansiedades de las personas (Atkinson y Blandy, 2016). Una de las razones es que justamente la vivienda es parte de un entorno amplio, y cualquier efecto de la diversidad del vecindario probablemente afecte la posición social de los residentes individuales (Brunton-Smith y Sturgis, 2011).

Hay investigaciones que sostienen que existen diferencias entre las viviendas que se han construido de manera formal, por medio de la gestión institucional, y las viviendas en asentamientos irregulares que se han gestionado informalmente a través de procesos de autoconstrucción (Aguirre, 2010). La literatura sobre este tema apunta a que la principal diferencia radica en el diseńo urbano y el acceso a los espacios públicos. $\mathrm{Al}$ respecto, Baena y Olaya (2013) enfatizan que al combatir la informalidad de la vivienda concluyen también los problemas de la percepción de inseguridad.

También hay evidencia sobre el origen público o comercial de la vivienda. En una investigación realizada en Shanghai, China, se encontró que los residentes de viviendas públicas -que posteriormente fueron transferidos a propiedad privada- se sentían menos seguros que los que vivían en viviendas comerciales (Yip, 2012).

Asimismo, diferentes tipos de construcción de la vivienda tienen efectos en relación con la inseguridad, de manera tal que los estratos medios y altos se concentran en conjuntos o fraccionamientos cerrados en busca de seguridad, mientras que las clases sociales medias y bajas se ubican en las viviendas producidas institucionalmente o autoconstruidas, lo cual evidencia que ambos estratos sociales se enfrentan de distintas maneras a la inseguridad (Aguirre, 2010). 
En mayor medida se han realizado análisis sobre viviendas en comunidades cerradas que proliferan en la mayoría de las ciudades del mundo (Newman, 1973; Blakely y Snyder, 1997; Caldeira, 2007; Davis, 2001; Bonvalet y Dureau, 2002; Grant y Mittelsteadt, 2004; López, 2008; Vilalta, 2013; Atkinson y Blandy, 2016). Pero también hay estudios que contrastan otras características de las viviendas.

Este tipo de diseño se asocia a la teoría del espacio defendible por Newman (1973), cuya propuesta arquitectónica apuesta por ambientes de viviendas, en los cuales la estructura física inhiba el delito al mostrar a los potenciales agresores que se trata de una comunidad que se defiende a sí misma.

Este tipo de comunidades se definen como un área residencial que está cercada y tiene control para el acceso y la salida al área de la vivienda (Abdullah et al., 2012; Caldeira, 2007). Son áreas residenciales con acceso restringido, en las que normalmente los espacios públicos son privatizados (Blakely y Snyder, 1997). Aunque también son "residencias, producidas como tal o resultantes del encerramiento de manzanas de casas individuales preexistentes" (Bonvalet y Dureau, 2002: 79).

En cuanto a los conjuntos o fraccionamientos cerrados, que principalmente se definen por la posesión de barreras físicas a la entrada de las viviendas, los hallazgos sobre la sensación de inseguridad no son conclusivos.

En distintas partes del mundo este fenómeno se ha intensificado, y en Estados Unidos el número de comunidades cerradas ha aumentado dramáticamente, como lo documentaron Blakely y Snyder (1997). Este fenómeno también se ha replicado en otras regiones como en América Latina, donde las comunidades cerradas de diferentes tipos y dimensiones son elementos comunes en todas las ciudades (Coy, 2006). Tal es el caso de México, donde la seguridad es la principal razón para decidir habitar o construir una comunidad cerrada o protegida (Guerrien, 2005).

En el contexto latinoamericano, los conjuntos residenciales cerrados están mayoritariamente habitados por personas con niveles socioeconómicos medio-altos y altos y, en general, se trata de ambientes bastante homogéneos, lo que los distingue de otras regiones del mundo donde no hay tal homogeneidad (Martínez, 2014). Aunque recientemente refieren los que estudian este fenómeno que este prototipo se ha convertido en un "nuevo modelo de habitar, valorado y reproducido también en los estratos medios y pobres" (Bonvalet y Dureau, 2002: 79).

En México, una parte del diseño urbano protegido se divide en dos grandes subconjuntos: los espacios residenciales protegidos, en donde principalmente habitan las personas con mayores ingresos económicos y sus respectivos espacios de vida urbana como las escuelas privadas, clubes deportivos, entre otros espacios diseñados para la misma población 
(Guerrien, 2005). Pero también hay zonas de seguridad (Blakely y Snyder, 1997) que fueron cerradas posteriormente por sus propios habitantes con diferentes tipos de barreras físicas o de vigilancia. Asimismo, hay condominios horizontales cuyo diseño fortificado se podría describir como bunkers residenciales (Guerrien, 2005).

Existen distintos tipos de comunidades cerradas, pero la mayoría se realiza con el fin de mejorar las condiciones de seguridad a nivel local y para huir del miedo y evadir el peligro que ocurre afuera (Grant y Mittelsteadt, 2004). En general "prometen la posibilidad de una vida cotidiana mejor, más armónica y protegida de la criminalidad” (López, 2008: 123). Lo que se espera es que el diseño de comunidades cerradas reduzca los actos delictivos en las áreas residenciales y esto mitigue la sensación de inseguridad (Blakely y Snyder, 1997). La expectativa del encerramiento es tal que incluso se incrementa el valor de los inmuebles (Davis, 2001).

En relación con las viviendas para las clases sociales medias y bajas, en la investigación realizada por Rollwagen (2016), los resultados sugieren que habitar en viviendas de varias unidades no tiene un impacto estadísticamente significativo en la sensación de seguridad. En el caso mexicano, Oehmichen (2013) realizó una investigación cualitativa en una unidad habitacional en la Ciudad de México; como parte de los hallazgos reporta que nueve de 10 entrevistados habían sido víctimas del delito -principalmente de asaltos- y entre las causas que atribuyen a la inseguridad en la colonia se encuentra el consumo y tráfico de drogas, y en cuanto a la sensación de inseguridad, refieren que el miedo se sufre individualmente, pero es socialmente compartido.

Mientras en algunas investigaciones se ha evidenciado que estas comunidades cerradas no son una solución consistente para controlar los niveles de miedo al crimen (Vilalta, 2013), en otras, los resultados reflejan que los residentes que habitan en áreas cerradas tienen un menor temor al crimen en comparación con aquellos que no tienen este tipo de fortificaciones (Blakely y Snyder, 1997). En el caso del amurallamiento, se arguye que hay un efecto fortaleza en el que las personas se sienten más seguras por tener una distancia del resto del vecindario (Rollwagen, 2016).

En dos ciudades de Malasia, al estudiar la forma típica de vivienda en las comunidades cerradas, en comparación con las viviendas sin fortificaciones - un concepto de desarrollo relativamente nuevo- se encontró que la sensación de inseguridad es mayor en las áreas residenciales cerradas y el resultado se atribuye principalmente a las relaciones en el ámbito comunitario (Abdullah et al., 2012).

En China, el estudio de Yip (2012) sobre las comunidades semicerradas en Shanghai-que se ha configurado como un simbolo de la vida modernase encontró que la sensación de inseguridad está estadísticamente relacionada 
con el grado de bloqueo. De manera tal que los barrios que no tenían muros mostraron niveles más bajos de percepción de seguridad, mientras que los vecindarios con control de acceso mostraron los niveles más altos. En contraparte, en Valledupar, Colombia, la investigación de Martínez (2014) muestra que en estas comunidades cerradas persiste la sensación de miedo, sobre todo en relación con los espacios cercanos, calificados como peligrosos.

En Tijuana, México, las viviendas con diseños fortificados son producto de procesos mundiales con matices locales (López, 2008) en los que existen variaciones, tanto físicas en cuanto a la composición social, pero en los que prevalece la idea de la relación entre el encierro y la seguridad que ha sido promovida y capitalizada por los desarrolladores inmobiliarios.

La configuración de estas comunidades cerradas tiene distintos efectos sociales, que a su vez podrían incidir en la sensación de inseguridad en la colonia. Uno de éstos es el aislamiento, donde "los vínculos sociales con el vecino interno están caracterizados por un nivel básico de encuentros esporádicos y fortuitos" (Martínez, 2014: 201), lo cual reduce la posibilidad de cohesión social, particularmente porque cuando la gente no conoce quiénes son sus vecinos se sienten más vulnerables (Body-Gendrot, 2008).

$\mathrm{Al}$ mismo tiempo, sus puertas y paredes reflejan el miedo y sirven como recordatorios diarios de los peligros percibidos en el otro lado (Blakely y Snyder, 1997). Y en conjunto, estas viviendas fortificadas modifican espacialmente las ciudades e impactan en las rutinas diarias de aquellos que habitan espacios segregados protegidos por muros, sistemas de vigilancia y acceso restringido (Caldeira, 2007) así como también afecta a quienes ahí transitan.

En muchos casos, las personas que viven en casas fortificadas desconocen la verdadera efectividad para reducir el miedo al delito (Vilalta, 2012), sobre todo en aquellos casos en los que el miedo no desaparece (López, 2008) y tampoco se desvanece la latente posibilidad de intrusiones aleatorias o violentas al hogar, que está en el centro de la preocupación de las personas (Atkinson y Blandy, 2016) porque incluso en estas comunidades ocurren delitos como robos y secuestros (Blakely y Snyder, 1997).

\subsection{Sobre las colonias}

El contexto de la colonia es una dimensión territorial de análisis relevante para el estudio de la sensación de la inseguridad y que se define como un lugar determinado por un conjunto específico de características basadas en el espacio que se encuentra en una escala geográfica concreta (Lebel et al., 2007). Esta dimensión de análisis es primordial para la comprensión de los lugares en la prevención del delito (Eck y Weisburd, 2015). 
Una colonia se integra de distintos componentes, entre los que se encuentran las viviendas que tienen una función fundamental en relación con la seguridad subjetiva. Se supone que están diseñadas para proporcionar sensación de seguridad a las personas dentro de su vecindario (Rollwagen, 2016). Y en comparación con otros componentes de la colonia, la vivienda ha logrado su cometido al configurarse -en las encuestas de victimización- como uno de los lugares en donde las personas se sienten más seguras en relación con otros lugares como el transporte público, el mercado, la calle o el parque.

En la colonia, la sensación de inseguridad está influida por la percepción de las colonias vecinas debido a su proximidad espacial, puesto que lo ocurre en una influye en la otra (Lebel et al., 2007). Al respecto, se ha encontrado evidencia de que los niveles promedio de miedo al crimen en los vecindarios cercanos se correlacionan significativamente (Wyant, 2008). También atañe a la variable tiempo, en donde la evidencia empírica indica que las personas que han vivido durante un periodo largo en el vecindario son más propensas a sentirse inseguros que los nuevos residentes (Rollwagen, 2016).

Se sabe que las diferencias individuales en la sensación de inseguridad están fuertemente relacionadas por las características socioeconómicas de la colonia, de manera tal que el miedo expresado al delito mejora en relación con las características de los entornos en los que viven (BruntonSmith y Sturgis, 2011). Así, hay un conjunto de variables sociales compartidas entre los habitantes de un espacio territorial e incluso se ha planteado que existe una cultura local de la inseguridad (Kessler, 2006).

Esta sensación varía considerablemente en relación con el entorno construido en términos del diseño residencial, particularmente respecto a la prevención del delito a través del diseño ambiental (CPTED) que, se ha evidenciado, tiene un efecto más fuerte al influir en la sensación de inseguridad que otras formas de prevención (Sakip et al., 2018). En contraparte, hay aspectos espaciales que contribuyen a la estigmatización de un barrio como la propia ubicación física, la precarización de la vivienda y el bajo valor económico de la tierra (Pyszczek, 2012).

También la sensación de inseguridad externa sobre la colonia se configura a partir de una evaluación que hacen las personas que no radican en ésta -con la información disponible-que tiene efectos directos en la configuración del vecindario. Los resultados de la investigación en Suecia de Ceccato y Wilhelmsson (2011) muestran que si el miedo y el vandalismo aumentan en la colonia se espera que los precios de los inmuebles disminuyan; con un precio implícito más alto para el miedo. En este sentido, las características de los vecindarios son determinantes fundamentales de cómo las personas perciben su riesgo ante la inseguridad 
en la colonia, aunque la evidencia empírica es inconsistente (BruntonSmith y Sturgis, 2011).

Pero además de estos elementos, que podrían ser evaluados interna y externamente, se refiere que hay un estigma del barrio, como una zona insegura que se mantiene y se retroalimenta y, a su vez, esto tiene distintos efectos como la segregación de los espacios, la menor inversión en comercios y viviendas, entre otros (Pyszczek, 2012). Es decir, se contribuye a mantener el círculo de la estigmatización y la sensación de inseguridad.

$\mathrm{El}$ incremento de los precios, en relación con las condiciones locales, corresponde al hecho de que las personas valoran mucho la seguridad de un vecindario cuando eligen un lugar para vivir (Ceccato y Wilhelmsson, 2011). Implica que el poder adquisitivo sea una restricción para poder vivir en entornos más seguros, de tal manera que la sensación de seguridad se asocia como una condicionante de la calidad de vida en las viviendas a la que tienen acceso los estratos socioeconómicos más altos, cuyos barrios presentan menos espacios deteriorados que se relacionen con percepciones de inseguridad (Gélvez, 2018).

También se ha encontrado que la satisfacción con el barrio es la variable que más explica la conformidad en general, seguida de la anuencia con la vivienda, y finalmente, en relación con los vecinos (Amérigo y Aragonés, 1990). Pero la vivienda en sí misma también tiene características que brindan o no seguridad a las personas que en ella habitan; por ejemplo, hay investigaciones que afirman que el tamaño de las construcciones de vivienda influye en el crimen, así como en el miedo al crimen (Newman y Franck, 1982). En suma, no es claro que los diferentes tipos de vivienda brinden sensación de seguridad de la misma manera (Rollwagen, 2016).

Esta condición de victimización por un alto número de habitantes en la colonia es relevante, porque la evidencia muestra que haber sido víctima de un delito en los últimos meses incrementa la sensación de inseguridad en cuanto a la perspectiva de un nuevo evento, respecto a quienes no lo han sido recientemente (Bergman y Kessler, 2008; Restrepo y Moreno 2007; Ruiz, 2007).

Cabe advertir que el robo a casa habitación es uno de los delitos que mayor temor generan entre las personas (Muratori y Zubieta, 2013; Atkinson y Blandy, 2016), no sólo por la pérdida de objetos materiales, sino también por el posible atentado a la integridad física de las personas que pudieran ser atacadas. En algunas investigaciones se ha mostrado que el efecto cambia de acuerdo con las distintas regiones de la ciudad, donde unas se consideran más peligrosas que otras (Sookram et al., 2011), pero en general, se ha encontrado un efecto positivo asociado con la experiencia del robo a casa habitación, tanto para el miedo específico a este delito, 
como en relación con la sensación de inseguridad en la colonia (Rountree y Land, 1996).

La sensación de inseguridad también está influida por otras variables como los medios de comunicación que cada vez son más próximos a los habitantes de las colonias y les permite compartir las noticias locales sobre violencia e inseguridad. Además de que, como lo han estudiado Atkinson y Blandy (2016), los nuevos medios se enfocan en la difusión de eventos que propagan la sensación de inseguridad al estar en la vivienda.

\section{Metodología}

\subsection{Fuente de información}

La fuente de información estadística para este estudio es la Encuesta Nacional de Victimización y Percepción sobre Seguridad Pública (ENVIPE) de 2019, organizada y levantada por el Instituto Nacional de Estadística y Geografía (Inegi) de México. Ésta es una encuesta de diseño transversal y probabilística compleja (trietápica, estratificada y por conglomerados) que se realiza anualmente desde el 2010 y que ofrece una representatividad en los resultados en los ámbitos nacional, nacional urbano, nacional rural, estado y para un conjunto de áreas metropolitanas del país. Es una encuesta en hogares con entrevistas cara a cara a la población adulta (mayores de 18 años). El levantamiento fue realizado entre el 1 de marzo y el 30 de abril del 2019. La muestra para ese año comprende un total de 102,043 viviendas. Las preguntas se refieren a eventos criminológicos sucedidos entre enero y diciembre de 2018 y a percepciones del estado de la seguridad pública y el desempeño de las autoridades al momento de realizar la encuesta.

\subsection{Variable dependiente}

La sensación de inseguridad en la colonia se mide de manera dicotómica, tal y como lo realiza la fuente de información de este estudio, la cual pregunta de forma directa al adulto encuestado lo siguiente: “¿En términos de delincuencia, considera que vivir en (ámbito geográfico) es... seguro (1), inseguro (2), no sabe/no responde (3)". Los diferentes ámbitos geográficos son tres: colonia o localidad, municipio o delegación o estado. Aquí nos enfocamos en el ámbito de la colonia o localidad. La opción tres de respuesta fue eliminada para el análisis. 


\subsection{Variables independientes}

Este estudio hace uso de dos variables independientes, las cuales son el tipo de vivienda (X1) y la barrera física a la entrada (X2). El tipo de vivienda (X1) se mide de manera dicotómica haciendo una distinción entre casa sola y edificio de departamentos. No obstante, existen tres categorías más de esta variable en la fuente de información, éstas son vivienda en vecindad, vivienda en cuarto de azotea y local no construido para habitación. Estas categorías no fueron incluidas en el análisis para efectos de mantener el estudio de la relación entre tipo de vivienda y sensación de inseguridad lo más claro y simple posible.

La posesión de una barrera física (X2) se mide también de manera dicotómica, pero en este caso desde su captura en la encuesta, al pedirle al encuestador que "identifique si la vivienda tiene alguna barrera física, como caseta de vigilancia, puertas con o sin intercomunicación de audio o video, portero u otra condición que le impida llegar de forma directa a la puerta de la vivienda". Las opciones de respuesta son... "si existe (1), no existe (2)".

En este sentido, cabe mencionar que ambas variables independientes son respondidas por el encuestador, no por el encuestado. Es decir, son apreciaciones visuales del encuestador previas a la realización de la encuesta. También cabe comentar que los tipos de barreras físicas pueden, por definición, ser de muchos tipos. En este caso, la fuente de información no permite conocer a detalle tales tipos; no obstante, su medición general nos permite saber que en la muestra nacional sólo una minoría de los adultos en el país poseen en sus viviendas algún tipo de barrera física entre la calle y la puerta de entrada a la misma (ver cuadro 1).

\section{Cuadro 1}

Frecuencias relativas en el cruce de las variables independientes

\begin{tabular}{lccc}
\hline & Sin barrera fisica & Con barrera fisica & Total \\
\hline Casa sola & $88.6 \%$ & $7.1 \%$ & $95.7 \%$ \\
Departamento & $1.6 \%$ & $2.7 \%$ & $4.3 \%$ \\
Total & $90.2 \%$ & $9.8 \%$ & $100.0 \%$ \\
\hline
\end{tabular}

$\mathrm{N}=89,951$

Fuente: elaboración propia con datos de la Envipe 2019 (Inegi, 2019). 


\subsection{Variables de control}

La selección de las variables de control se hizo acorde a los hallazgos en estudios anteriores. De esta manera, se seleccionaron variables representativas de la teoría de vulnerabilidad física, vulnerabilidad social, incivilidades, victimización y redes sociales (ver cuadro 2 ).

\section{Cuadro 2}

Teorías y variables de control

\begin{tabular}{lll}
\hline \multicolumn{1}{c}{ Teoría } & \multicolumn{1}{c}{ Variable de control } & \multicolumn{1}{c}{ Medición } \\
\hline Vulnerabilidad física & Mujer & Dicotómica $(\mathrm{No}=0, \mathrm{Si}=1)$ \\
& Edad & Continua $(18$ a 97) \\
& Edad cuadrática & Continua \\
Vulnerabilidad social & Estudios universitarios & Dicotómica $(\mathrm{No}=0, \mathrm{Si}=1)$ \\
Victimización & Victimización previa & Dicotómica $(\mathrm{No}=0, \mathrm{Si}=1)$ \\
Incivilidades & Señales de desorden social & Dicotómica $(\mathrm{No}=0, \mathrm{Si}=1)$ \\
Redes sociales & Tiempo de residencia $(>1$ año $)$ & Dicotómica $(\mathrm{No}=0, \mathrm{Si}=1)$ \\
& Confianza en los vecinos & Dicotómica $(\mathrm{No}=0, \mathrm{Si}=1)$ \\
& Percepción de corrupción policial & Dicotómica $(\mathrm{No}=0, \mathrm{Si}=1)$ \\
\hline
\end{tabular}

Fuente: elaboración propia con datos de la Envipe 2019 (Inegi, 2019).

Diversas variables de control fueron recalculadas para representarse dicotómicamente; por ejemplo, la escolaridad fue dividida en dos posibles categorías: $\sin /$ con estudios universitarios. Ésta es una variable representativa de vulnerabilidad social, ya que un grado universitario se correlaciona con un mayor ingreso, lo cual teóricamente reduce el impacto económico que puede tener la experiencia de una victimización delictiva. Por lo anterior, se asume que menor vulnerabilidad social corresponde con una menor sensación de inseguridad.

$\mathrm{Al}$ respecto de las señales de desorden social como representativa de la teoría de incivilidades, la encuesta mide 15 diferentes situaciones problemáticas que suceden alrededor de la vivienda, y que varían desde consumo de alcohol en la calle o riñas entre vecinos, hasta el reporte de secuestros y homicidios. En este caso, por simpleza de análisis, se redujeron las categorías de desorden social a dos posibilidades de respuesta: no hay situaciones de desorden (0) o sí hay situaciones de desorden (1).

La experiencia de victimización previa puede abarcar cualquier tipo de delito del que los encuestados hayan sido víctimas el año o los años 
anteriores. El tiempo de residencia se mide en la encuesta con cuatro posibilidades de respuesta: "menos de seis meses (1), entre seis meses y un año (2) o más de un año (3)". En este estudio decidimos reducir la variable a un nivel dicotómico para representar menos de un año (0) o más de un año (1). El grado de confianza en los vecinos se mide en la encuesta de manera ordinal: "mucha (1), alguna (2), poca (3), nada (4)". En este estudio redujimos la variable a un nivel dicotómico, agrupando las categorías uno a tres como sí tiene confianza (1) y la cuatro en no tiene confianza (0). Finalmente, la percepción de corrupción de policía local también tiene un nivel dicotómico desde la realización de la encuesta, en donde se le pregunta al encuestado: "a su juicio ¡la (autoridad) puede calificarse como corrupta? En este caso sólo existen tres opciones de respuesta: "si (1), no (2), no sabe/no responde (3)". Se utilizó como autoridad de referencia a la policía preventiva municipal para el caso de todos los estados y la policía de la Ciudad de México, siendo que esta última no posee policías municipales. Naturalmente, las opciones de respuesta "no aplica, no sabe/no responde", en cualquiera de las variables de control, no fueron consideradas en el análisis.

\subsection{Análisis estadístico}

Se utilizaron tres modelos de regresión logística binaria multinivel para probar la asociación estadística entre el tipo de vivienda, la presencia de una barrera física y su interacción, con la sensación de inseguridad en la colonia, controlando la covariación de diferentes correlativos teóricos en pasos diferentes, iniciando desde un modelo nulo (sin variables independientes ni correlativos) hasta un modelo completo. Esto llevó a tener un total de seis modelos resultantes. Los resultados de este modelo logístico se confrontaron con estimaciones provenientes de modelos probit equivalentes, sobre los cuales no se vieron diferencias sustantivas en los resultados. Adicionalmente se utilizaron errores robustos estándar para reducir las afectaciones que una posible heterocedasticidad tendría en las pruebas de significancia.

La estructura multinivel representa la agrupación jerárquica de los datos transversales, siendo el nivel 1 el encuestado y el nivel 2 el estado de residencia. Se obtuvieron los coeficientes de correlación intraclase (ICC) en cada modelo. Por simpleza de interpretación de los resultados se presentan las razones de momios (OR) de los coeficientes. Se calculó el criterio de información de Akaike (AIC) para comparar la calidad de ajuste de los datos entre modelos. Los análisis se realizaron con el uso del paquete Stata v. 15.1 (Stata Corp, 2017). 


\section{Resultados}

Como se muestra en el cuadro 3, alrededor de 53\% de los residentes en departamentos reportan sentirse inseguros en sus colonias, frente a $46 \%$ de los residentes en casas solas. Igualmente, aquellos con barreras físicas a la entrada de sus viviendas que reportan sentirse inseguros en sus colonias son proporcionalmente más (49\%) que aquellos que no poseen algún tipo de barrera física a la entrada de la vivienda (46\%). También se puede observar una mayor proporción de adultos que reportaron sentirse inseguros en sus colonias entre aquellos que viven en departamentos con barreras físicas (53\%) frente a su contraparte, es decir, aquellos que viven en casas solas sin barreras físicas a la entrada (46\%). Todas las diferencias anteriores son estadísticamente significativas. Estas diferencias dejan de tener significancia estadística una vez que son consideradas dentro de los modelos multivariados que mostramos a continuación.

\section{Cuadro 3}

Sensación de inseguridad en la colonia, tipo de vivienda y posesión de barrera física a la entrada de la vivienda (Envipe, 2019)

\begin{tabular}{lccc}
\hline & Seguro & Inseguro & $\begin{array}{c}\text { Chi-cuadrado de } \\
\text { Pearson }\end{array}$ \\
\hline Casa sola & $53.9 \%$ & $46.1 \%$ & $78.8^{* * *}$ \\
Departamento & $46.6 \%$ & $53.4 \%$ & \\
\hline Sin barrera física & $53.7 \%$ & $46.3 \%$ & $30.7^{* * *}$ \\
Con barrera física & $50.7 \%$ & $49.3 \%$ & \\
\hline Casa sola sin barr. fís. & $53.8 \%$ & $46.2 \%$ & $44.1^{* * *}$ \\
Departamento con barr. fís. & $46.9 \%$ & $53.1 \%$ & \\
\hline
\end{tabular}

*** $\mathrm{p}<0.01,{ }^{* *} \mathrm{p}<0.05,{ }^{*} \mathrm{p}<0.10$

Fuente: elaboración propia con datos de la Envipe 2019 (Inegi, 2019).

El cuadro 4 presenta los resultados de los diferentes modelos de regresión logística multinivel, estimando las correlaciones independientes entre el tipo de vivienda y la presencia de barreras físicas a la entrada de la misma con la sensación de inseguridad en la colonia. Las pruebas de significancia se realizaron con errores estándar robustos. Las estimaciones multinivel alternativas bajo diferentes suposiciones, como las basadas en la estimación probit también con soluciones por máxima verosimilitud, 


\section{Cuadro 4}

\section{Resultados de la regresión logística multinivel (OR)}

\begin{tabular}{|c|c|c|c|c|c|c|}
\hline & Modelo (1) & Modelo & Modelo (3) & Modelo & Modelo (5) & Modelo (6) \\
\hline Departamento (X1) & & 1.054 & 1.082 & 1.042 & 1.040 & 1.000 \\
\hline & & $(0.075)$ & $(0.077)$ & $(0.073)$ & $(0.072)$ & $(0.075)$ \\
\hline Barrera física (X2) & & 1.207 & 1.232 & 1.181 & 1.213 & 0.990 \\
\hline & & $(0.278)$ & $(0.293)$ & $(0.277)$ & $(0.279)$ & $(0.278)$ \\
\hline Interacción & & 0.700 & 0.693 & 0.713 & 0.720 & 0.833 \\
\hline & & $(0.158)$ & $(0.162)$ & $(0.163)$ & $(0.161)$ & $(0.222)$ \\
\hline Mujer & & & $1.431^{* * *}$ & $1.449^{* * *}$ & $1.464^{* * *}$ & $1.489^{* * *}$ \\
\hline & & & $(0.031)$ & $(0.031)$ & $(0.033)$ & $(0.034)$ \\
\hline Edad & & & $1.042^{* * *}$ & $1.042^{* * *}$ & $1.040^{* * *}$ & $1.037^{* * *}$ \\
\hline & & & $(0.003)$ & $(0.003)$ & $(0.003)$ & $(0.004)$ \\
\hline Edad cuadrática & & & $1.000^{* * *}$ & $1.000^{* * *}$ & $1.000^{* * *}$ & $1.000^{* * *}$ \\
\hline & & & $(0.000)$ & $(0.000)$ & $(0.000)$ & $(0.000)$ \\
\hline Estudios & & & $0.889^{* *}$ & $0.781^{* * *}$ & $0.781^{* * *}$ & $0.773^{* * *}$ \\
\hline & & & $(0.048)$ & $(0.039)$ & $(0.035)$ & $(0.034)$ \\
\hline Resid. colonia & & & & $1.988^{* * *}$ & $1.805^{* * *}$ & $1.705^{* * *}$ \\
\hline & & & & $(0.043)$ & $(0.037)$ & $(0.038)$ \\
\hline Victimización & & & & & $3.967^{* * *}$ & $4.148^{* * *}$ \\
\hline & & & & & $(0.211)$ & $(0.222)$ \\
\hline Señales de desorden & & & & & & $1.165^{* * *}$ \\
\hline & & & & & & $(0.037)$ \\
\hline Confianza en & & & & & & $0.557^{* * *}$ \\
\hline & & & & & & $(0.021)$ \\
\hline Corrupción & & & & & & $1.566^{* * *}$ \\
\hline & & & & & & $(0.043)$ \\
\hline Constante & $0.846^{* *}$ & $0.861^{*}$ & $0.300^{* * *}$ & $0.231^{* * *}$ & $0.068^{* * *}$ & $\quad 0.070^{* * *}$ \\
\hline & $(0.066)$ & $(0.070)$ & $(0.034)$ & $(0.026)$ & $(0.008)$ & (0.008) \\
\hline Var(constante) & 0.185 & 0.197 & 0.199 & 0.173 & 0.151 & 0.145 \\
\hline & $(0.051)$ & $(0.060)$ & $(0.061)$ & $(0.051)$ & $(0.043)$ & $(0.037)$ \\
\hline
\end{tabular}


Cuadro 4 (continuación)

\begin{tabular}{lcccccc}
\hline & Modelo (1) & $\begin{array}{c}\text { Modelo } \\
(2)\end{array}$ & Modelo (3) & $\begin{array}{c}\text { Modelo } \\
\text { (4) }\end{array}$ & Modelo (5) Modelo (6) \\
\hline ICC & 0.053 & 0.056 & 0.057 & 0.500 & 0.044 & 0.042 \\
\hline AIC & 122,014 & 119,352 & 117,572 & 115,150 & 111,980 & 65,444 \\
\hline $\begin{array}{l}\text { Chi-cuadrado } \\
\text { de Wald }\end{array}$ & n.a. & $13.5^{* * *}$ & $700.1^{* * *}$ & $2046.7^{* * *}$ & $2089.8^{* * *}$ & $4040.2^{* * *}$ \\
\hline Obs. & 91,527 & 89,597 & 89,058 & 88,742 & 88,742 & 52,441 \\
\hline Grupos & 32 & 32 & 32 & 32 & 32 & 32 \\
\hline
\end{tabular}

Errores estándar robustos en paréntesis

*** $\mathrm{p}<0.01,{ }^{* *} \mathrm{p}<0.05,{ }^{*} \mathrm{p}<0.1$

Fuente: elaboración propia con datos de la Envipe, 2019 (Inegi, 2019).

producen los mismos resultados sustantivos. Se muestran las razones de momios (OR) por simpleza de interpretación.

Los resultados indican que los residentes en departamentos no poseen una proclividad significativamente diferente de los residentes en casas solas a reportarse inseguros en su colonia de residencia. Igualmente, aquellos que poseen barrera(s) física(s) a la entrada de su vivienda tampoco poseen una proclividad diferente, mayor o menor, a reportar una sensación de inseguridad en su colonia de residencia. A la vez, el término de interacción entre ambas variables tampoco hace una diferencia en la proclividad a reportar un estado de inseguridad. En este sentido, ni el tipo de vivienda ni una barrera física colocada a la entrada, así como una combinación de las circunstancias anteriores, tiene algún efecto en la sensación de inseguridad en la colonia. Ambas son estadísticamente inconsecuentes para predecir la sensación de inseguridad en la colonia.

Entre las variables de control, observamos varias correlaciones de magnitud estadística e importancia teórica y de política pública. Por ejemplo, vemos el fuerte impacto que provocan las señales de desorden social en la colonia en la sensación de inseguridad en ese mismo espacio. El coeficiente del modelo completo (modelo 3) indica que un encuestado que reside en una colonia con señales de desorden social es hasta tres veces (314\%) más proclive a sentirse inseguro que su contraparte, es decir, aquel que no reporta vivir en una colonia con seńales de desorden social. Igualmente, la experiencia de haber sido víctima de un delito (o varios) incrementa la proclividad a sentirse inseguro: $70 \%$, aproximadamente.

La percepción de que la policía local es corrupta también exacerba la sensación de inseguridad en la colonia. En ese caso, aquellos con esa percepción son $56 \%$ más proclives a reportar sentirse inseguros en su 
colonia. Las mujeres son $48 \%$ más proclives a reportar una sensación de inseguridad en su colonia frente a los hombres. Igualmente, aquellos que llevan más de un año residiendo en la colonia reportan tal inseguridad con una mayor proclividad que aquellos que reportan que llevan menos de un año.

En cambio, aquellos que confían en los vecinos tienen una menor proclividad a sentirse inseguros (44\% menos), así como aquellos con estudios universitarios ( $23 \%$ menos). La edad es un correlativo especial de la sensación de inseguridad en cuanto a que nuestros resultados indican, de manera semejante a estudios previos, que conforme aumenta la edad también aumenta la proclividad a sentirse inseguro en la colonia, pero hasta cierto momento en que la tendencia se vuelve opuesta; a mayor edad, menor sensación de inseguridad.

Finalmente, los coeficientes de correlación intraclase (ICC) de cada modelo indican que la localización de los encuestados en cada estado explica entre $5.6 \%$ y $4.2 \%$ la varianza en la sensación de inseguridad en la colonia, dependiendo del número de correlativos o variables de control incluidas en los modelos. Las estadísticas descriptivas del modelo utilizado se muestran en el anexo, cuadro $1 \mathrm{~A}$.

\section{Discusión y conclusiones}

Los hallazgos de este estudio sugieren que vivir en una casa sola o un edificio de departamentos no determina la sensación de inseguridad en la colonia. Igualmente, la barrera física a la entrada de la vivienda tampoco hace una diferencia para determinar si un adulto se siente inseguro en su colonia de residencia. Finalmente, la combinación de tipo de vivienda y barrera física tampoco determinan la sensación de inseguridad en la colonia.

Iniciando este estudio argumentamos que el tipo de vivienda y las barreras físicas colocadas entre la entrada y la calle podrían tener alguna correlación con la sensación de inseguridad de los vecinos en la colonia. La lógica de nuestro argumento se basó en que son las personas que se sienten más inseguras en sus colonias las que vivirían en tipos o diseños de viviendas que ofrecen una mayor protección frente al riesgo de victimización delictiva. La premisa es que son aquellos con más sensación de inseguridad en su entorno inmediato, los que viven más aislados o protegidos del mismo. En este sentido, pensamos que los residentes en edificios de departamentos reportarían mayores niveles de sensación de inseguridad en sus colonias en comparación con los residentes en casas solas. Igualmente, consideramos que aquellos residentes, ya fuera en departamentos o casas solas que tuvieran algún tipo de barrera física entre 
la entrada a la vivienda y la calle, también tendrían una mayor proclividad a reportarse inseguros en su colonia. Por deducción, pensamos lo mismo entre aquellos residentes en viviendas que combinaran la circunstancia de vivir en un departamento y tuvieran además alguna barrera física a la entrada de su vivienda. Las pruebas bivariadas mostraron indicios estadísticos de tal circunstancia; no obstante, los modelos multivariados dejaron desde el principio la ausencia de evidencia sobre tal correlación. No hay una correlación estadísticamente significativa e independiente entre el tipo de vivienda y la posesión de alguna barrera física a la entrada de la misma.

Aunque el diseño de este estudio fue transversal en oposición a longitudinal, y para sólo una vuelta (la más reciente) de la encuesta de victimización y percepción de seguridad pública en México, la ausencia de evidencia al respecto de una correlación independiente entre el tipo de vivienda y las barreras físicas colocadas a la entrada de la misma se sostiene como válida y valiosa aun con las limitaciones de diseño del estudio. Un estudio con un diseño diferente no podría refutar nuestro hallazgo con respecto a que no existe evidencia en la correlación bajo estudio. Si acaso, podría refutar lo contrario: una correlación espuria o fortuita. La carga de la prueba reside en la hipótesis alternativa.

Nuestro estudio tiene otras limitaciones. El diseño dejó fuera a los residentes de viviendas en vecindades, en cuartos de azoteas y en locales no construidos para habitación, los cuales son en su totalidad 2.1\% de los encuestados. Esto se realizó para efectos de mantener simpleza en las pruebas de comparación entre tipos de viviendas, siendo las principales, por el amplio número de residentes en México, las casas solas y los departamentos en edificios, con $93.7 \%$ y $4.2 \%$ del total de encuestados. Por otro lado, si bien sabemos, a partir de la misma encuesta, si la vivienda poseía algún tipo de barrera física, no sabemos a cuál tipo se refiere. Por esta razón, nuestros resultados deben interpretarse como exploratorios en materia de medios de protección o aislamiento de las viviendas a su entorno de calle. Sin embargo, hasta que existan otras fuentes de información sobre los tipos de barreras físicas a la puerta de entrada de la vivienda, nuestra mejor opción sigue siendo la Envipe. Otras limitaciones del presente estudio es que nuestra evidencia es observacional en oposición a experimental o cuasi-experimental. Por lo tanto, los resultados son inherentemente sensibles a un sesgo de selección en la encuesta. Este posible riesgo no puede ser resuelto por los investigadores.

Otra limitación radica en nuestro uso del estado de residencia como una fuente de efectos contextuales. La limitación radica en que el espacio estatal no refleja necesariamente las divisiones funcionales o contextuales de los lugares de residencia de los encuestados. En todo caso, esta partición de la 
varianza por estado de residencia de los encuestados arrojó que efectivamente hay factores en ese ámbito espacial que agregan algún grado de variación en el reporte de sensación de inseguridad en la colonia. Posiblemente, siguientes estudios podrán hacer diferenciaciones entre zonas metropolitanas o municipios de residencia de los encuestados, para efectos de discernir mejor tales efectos contextuales.

Aun con las limitaciones ya mencionadas, este estudio muestra que la sensación de inseguridad en la colonia no parece estar condicionada ni por el tipo de vivienda ni por la presencia de barreras físicas entre la calle y la entrada a la misma. En cambio, por lo que sí está condicionada esta sensación de inseguridad es por las señales de desorden social en la colonia, la experiencia de una victimización delictiva previa, una percepción de que la policía local es corrupta, ser mujer, la desconfianza en los vecinos, el grado de escolaridad, el tiempo de residencia en la colonia y la edad, hasta cierta edad, en donde a mayor edad se reduce la proclividad a sentirse inseguro en la colonia. Estos hallazgos tienen implicaciones teóricas en el estudio de la relación entre nuestros estilos urbanísticos y la sensación de inseguridad. Igualmente, varios de estos hallazgos tienen implicaciones de política de seguridad pública. Queda claro que orientar la seguridad pública hacia aspectos de percepción de seguridad puede incrementar la calidad de vida. Reducir los niveles de desorden social y de percepción de corrupción policial puede aumentar significativamente sensación de seguridad y nuestra calidad de vida en México.

\section{Anexo}

Cuadro A1

Estadísticos descriptivos de la muestra de estimación del modelo completo (Modelo 6)

\begin{tabular}{lcccc}
\hline & Media & Desv. Est. & Min. & Max. \\
\hline Inseguridad en la colonia & 0.459 & 0.498 & 0 & 1 \\
Departamento (X1) & 0.039 & 0.195 & 0 & 1 \\
Barrera física (X2) & 0.092 & 0.289 & 0 & 1 \\
Interacción (X1*X2) & 0.116 & 0.387 & 0 & 2 \\
Mujer & 0.485 & 0.500 & 0 & 1 \\
Edad & 41.778 & 15.662 & 18 & 97 \\
Edad cuadrática & 1990.7 & 1474.0 & 324 & 9,409 \\
\hline
\end{tabular}


Cuadro A1 (continuación)

\begin{tabular}{lcccc}
\hline & Media & Desv. Est. & Min. & Max. \\
\hline Estudios univ. & 0.219 & 0.414 & 0 & 1 \\
Tiempo de resid. (>1 año) & 0.928 & 0.258 & 0 & 1 \\
Victimización prev. & 0.368 & 0.482 & 0 & 1 \\
Señales de desorden & 0.896 & 0.305 & 0 & 1 \\
Confianza en vecinos & 0.908 & 0.288 & 0 & 1 \\
Corrupción policial & 0.722 & 0.448 & 0 & 1 \\
\hline
\end{tabular}

Fuente: elaboración propia.

\section{Fuentes consultadas}

Abdullah, Aldrin; Mohd Salleh, Mohd Najib y Md. Sakip, Siti Rasidah (2012), "Fear of crime in gated and non-gated residential areas", Procedia-Social and Behavioral Sciences, vol. 35, Elsevier, pp. 63-69.

Aguirre, Edwin (2010), "Escenarios de violencia urbana. Usos y percepciones del espacio público relacionado con la vivienda en Ciudad Juárez, Chihuahua", tesis de maestría, El Colegio de la Frontera del Norte, Tijuana.

Amérigo, Maria y Aragonés, Juan Ignacio (1990), "Residential satisfaction in council housing”, Journal of Environmental Psychology, 10 (4), Elsevier, pp. 313-325.

Atkinson, Rowland y Blandy, Sarah (2016), Domestic fortress: Fear and the new home front, Manchester, Manchester University Press.

Baena, Andrea y Olaya, Camilo (2013), "Vivienda de interés social de calidad en Colombia: hacia una solución integral", Sistemas y Telemática, 11 (24), Cali, Universidad ICESI, pp. 9-26.

Bergman, Marcelo y Kessler, Gabriel (2008), "Vulnerabilidad al delito y sentimiento de inseguridad en Buenos Aires: Determinantes y consecuencias", Desarrollo Económico, 48 (190/191), Buenos Aires, Instituto de Desarrollo Económico y Social, pp. 209-234. 
Blakely, Edward y Snyder, Mary Gail (1997), Fortress America gated communities in The United States, Washington D. C., Brookings Institution Press.

Body-Gendrot, Sophie (2008), "Grandes evoluciones sociales en las áreas metropolitanas”, en Gimeno Ortiz de Urbina y Juli Ponce Solé (coords.), Convivencia ciudadana, seguridad pública y urbanismo. Diez textos fundamentales del panorama internacional, Madrid, Fundación Democracia y Gobierno Local y Diputació de Barcelona, pp. 69-88.

Bonvalet, Catherine y Dureau, Françoise (2002), "Los modos de habitar: unas decisiones condicionadas", en Françoise Dureau, Véronique Dupont, Éva Lelièvre, Jean-Pierre Lévy y Thierry Lulle (coords.), Metrópolis en movimiento. Una comparación internacional, Bogotá, Alfaomega, pp. 69-87.

Brunton-Smith, Ian y Sturgis, Patrick (2011), "Do neighborhoods generate fear of crime? An empirical test using the British crime survey", Criminology, 49 (2), Ohio, The American Society of Criminology, pp. 331-369.

Caldeira, Teresa (2007), City of walls: crime, segregation, and citizenship in São Paulo, Berkeley, University of California Press.

Ceccato, Vania y Wilhelmsson, Mats (2011), "Acts of vandalism and fear in neighbourhoods: do they affect housing prices?", en Vania Ceccato (ed.), The urban fabric of crime and fear, Springer, pp. 191-213.

Coy, Martin (2006), "Gated communities and urban fragmentation in Latin America: the Brazilian experience", GeoJournal, 66 (1/2), Springer, pp. 121-132.

Davis, Mike (2001), Más allá de Blade Runner. "Control urbano, la ecología del miedo”, Barcelona, VIRUS Editorial.

Eck, John y Weisburd, David (2015), "Crime places in crime theory", Crime and place: Crime prevention studies, vol. 4, Jerusalén, Hebrew University of Jerusalem Legal Research Paper, pp. 1-33.

Gélvez, Juan David (2018), “¿Cuáles determinantes se relacionan con la percepción de inseguridad? Un análisis estadístico y espacial para 
la ciudad de Bogotá, D. C.”, Revista Criminalidad, 61 (1), Bogotá, Dirección de Investigación Criminal, pp. 69-84.

Grant, Jill y Mittelsteadt, Lindsey (2004), "Types of gated communities", Environment and planning B: Planning and Design, 31 (6), Sage Journals, pp. 913-930.

Guerrien, Marc (2005), “Arquitectura de la inseguridad, percepción del crimen y fragmentación del espacio urbano en la zona metropolitana del valle de México", en Quim Bonastra, Celeste Arella, Gabriela Rodríguez Fernández y Pedro Fraile Pérez de Mendiguren (coords.) Paisaje ciudadano, delito y percepción de la inseguridad: investigación interdisciplinaria del medio urbano, Madrid, Dykinson, pp. 23-116.

Inegi (Instituto Nacional de Estadística y Geografía) (2019), "Encuesta Nacional de Victimización y Percepción sobre Seguridad Pública (Envipe) 2019. Principales Resultados”, Aguascalientes, Inegi.

Kessler, Gabriel (2006), El sentimiento de inseguridad. Sociología del temor al delito, Buenos Aires, Siglo XXI.

Lebel, Alexandre; Pampalon, Robert y Villeneuve, Paul (2007), "A multiperspective approach for defining neighborhood units in the context of health inequalities in the Quebec city region", International Journal of Health Geographics, 6 (1), Bio Med Central, Springer, pp. 1-15.

López Levi, Liliana (2008), “Tijuana: imaginarios globales, fortificaciones locales", Sociológica, 23 (66), Ciudad de México, UAM unidad Azcapotzalco, pp. 121-153.

Martínez, Hermes (2014), "Imaginarios sociales urbanos relacionados con los conjuntos residenciales cerrados en Valledupar", Katharsis, Revista de Ciencias Sociales, núm. 18, Envigado, Institución Universitaria de Envigado, pp. 191-210.

Muratori, Marcela y Zubieta, Elena Mercedes (2013), "Miedo al delito y victimización como factores influyentes en la percepción del contexto social y clima emocional", Boletín de Psicología, núm. 109, Valencia, Universidad de Valencia, pp. 7-18. 
Newman, Oscar (1973), Defensible space: Crime prevention through urban design, Nueva York, MacMillan Publishing Company.

Newman, Oscar y Franck, Karen (1982), "The effects of building size on personal crime and fear of crime", Population and Environment, 5 (4), Switzerland, Springer Nature, pp. 203-220.

Oehmichen, Cristina (2013), "La violencia de siempre: Representaciones de la violencia delincuencial en un barrio popular de la Ciudad de México", Anales de Antropología, 47 (1), Ciudad de México, UNAM/Instituto de Investigaciones Antropológicas, pp. 243-262.

Pyszczek, Oscar Luis (2012), "Los espacios subjetivos del miedo: construcción de la estigmatización espacial en relación con la inseguridad delictiva urbana”, Cuadernos de Geografía Revista Colombiana de Geografía, 21 (1), Bogotá, Universidad Nacional de Colombia, pp. 41-54.

Restrepo, Elvira María y Moreno, Álvaro José (2007), "Bogotá: ¿Más delito? ¿Más miedo?”, Desarrollo y Sociedad, núm. 59, Bogotá, Universidad de los Andes, pp. 165-214.

Rollwagen, Heather (2016), “The relationship between dwelling type and fear of crime", Environment and Behavior, 48 (2), Sage Journals, pp. 365-387.

Rountree, Pamela Wilcox y Land, Kenneth (1996), "Burglary victimization, perceptions of crime risk, and routine activities: A multilevel analysis across Seattle neighborhoods and census tracts", Journal of research in crime and delinquency, 33 (2), Sage Journals, pp. 147-180.

Ruiz Pérez, José Ignacio (2007), "Cultura Ciudadana, miedo al delito y victimización: un análisis de sus interrelaciones desde la perspectiva del tejido social", Acta Colombiana de Psicología, 10 (1), Bogotá, Universidad Nacional de Colombia, pp. 65-74.

Sakip, Siti Rasida; Johari, Noraini y Salleh, Mohd Najib Mohd (2018), "The Relationship of CPTED and Fear of Crime in Gated and Non-Gated Residential”, Asian Journal of Behavioural Studies, 3 (9), Sheffield, International Publishing House, pp. 195-205. 
Sookram, Sandra; Saridakis, George y Mohammed, Anne Marie (2011), "Do victims of crime fear crime more? Empirical evidence from the survey of living conditions (2005) of Trinidad and Tobago", Social and Economic Studies, 60 (2), Washington D. C., Council for Social and Economic Studies, pp. 127-144.

StataCorp (2017), "Stata Statistical Software", Release 15.1, College Station, TX: StataCorp LLC.

Vilalta, Carlos (2013), "La inefectividad de las comunidades cerradas para controlar el miedo al crimen", Gestión y Política Pública, volumen temático, Ciudad de México, Centro de Investigación y Docencia Económicas (CIDE), pp. 127-155.

Vilalta, Carlos (2012), "Fear of crime and home security systems", Police Practice and Research, 13 (1), Londres, Taylor \& Francis, pp. 4-14.

Wyant, Brian (2008), "Multilevel impacts of perceived incivilities and perceptions of crime risk on fear of crime", Journal of Research in Crime and Delinquency, 45 (1), Los Ángeles, Sage Publications Sage CA, pp. 39-64.

Yip, Ngai Ming (2012), "Walled without gates: Gated communities in Shanghai", Urban Geography, 33 (2), Londres, Taylor \& Francis, pp. 221-236.

Recibido: 27 de marzo de 2020. Reenviado: 10 de agosto de 2020. Aceptado: 20 de agosto de 2020.

Carlos Vilalta. Doctor en estudios urbanos, por Portland State University. Actualmente se encuentra adscripto al Centro de Investigación en Ciencias de Información Geoespacial, A. C. Es miembro del Sistema Nacional de Investigadores nivel III. Sus líneas de investigación son: elementos temporales y espaciales del crimen, miedo al crimen, estadística espacial, paramétrica, no-paramétrica y series de tiempo. Entre sus más recientes publicaciones se encuentran, como coautor: "Homicide as a function of city block layout: Mexico City as Case Study", Global Crime, 21 (2), Nueva York, Routledge, Taylor \& Francis, pp. 111-129 (2020); "Modeling crime in an uptown neighborhood: The case of Santa Fe in Mexico City", Papers in Applied Geography, 5 (1-2), Nueva York, Routledge, Taylor \& Francis, 
pp. 1-12 (2019), y "A descriptive model of the relationship between Police CCTV systems and crime. Evidence from Mexico City", Police Practice and Research, 20 (2), Nueva York, Routledge, Taylor \& Francis, pp. 105-121 (2018).

Lucía Carmina Jasso. Doctora en políticas públicas con énfasis en Análisis Organizacional e Institucional por el Centro de Investigación y Docencia Económicas, A. C. (CIDE). Actualmente labora en el Instituto de Investigaciones Sociales de la Universidad Nacional Autónoma de México (UNAM). Es miembro del Sistema Nacional de Investigadores nivel candidata. Sus líneas de investigación son: seguridad pública y políticas públicas para la reducción y prevención de la criminalidad. Entre sus más recientes publicaciones se encuentran, como autora: "Seguridad ciudadana y tecnología: uso, planeación y regulación de la videovigilancia en Latinoamérica", Dikê Revista de investigación en Derecho, Criminología y Consultoría Jurídica, núm. 27, Puebla, Benemérita Universidad Autónoma de Puebla, pp. 5-27 (2020); “Tecnologías de vigilancia en las empresas mexicanas para protegerse de la inseguridad", Hipertextos, 8 (14), Buenos Aires, Universidad de Buenos Aires (UBA) (próximamente); "La violencia feminicida y el feminicidio en la península de Yucatán: características y contextos regionales", Antrópica. Revista de Ciencias Sociales y Humanidades, 5 (10), Mérida, Universidad Autónoma de Yucatán, pp. 21-46 (2019), y "El rescate de espacios públicos en México: una aproximación a la política pública de prevención situacional del delito", Revista Criminalidad, 61 (1), Bogotá, Policía Nacional de Colombia, pp. 51-67 (2019); como coautora: "Transformación del Estado y privatización de la seguridad pública. Policías privadas, cárceles privadas y gated communities en México”, Ciudad de México, Universidad Nacional Autónoma de México/Instituto de Investigaciones Sociales (próximamente).

Gustavo Fondevila. Doctor en derecho por la Universidad de Buenos Aires (2001). Actualmente labora en el Centro de Investigación y Docencia Económica (CIDE). Es miembro del Sistema Nacional de Investigadores nivel III. Sus líneas de investigación se relacionan con el estudio del comportamiento del delito, victimización, miedo al crimen, variaciones en las tasas criminales y patrones delictivos; estudios policiales como actividades rutinarias y vigilancia policial en puntos críticos. Entre sus más recientes publicaciones se encuentran, como coautor: "Homicide as a function of city block layout: Mexico City as Case Study", Global Crime, 21 (2), Nueva York, Routledge, Taylor \& Francis, pp. 111-129 (2020); "Concentrated violence: the influence of criminal activity and governance on prison violence in Latin America", International Criminal Justice Review, 
30 (1), SAGE Publications, pp. 99-130 (2019), y “Convergence dynamics of robbery rate in Mexico", Crime \& Celinquency, 64 (14), SAGE Publications, pp. 105-121 (2018). 